\title{
ANÁLISIS CARTOGRÁFICO DE LA DIMENSIÓN FRACTAL PARA LA VARIACIÓN ESPACIO-TEMPORAL DE RÍOS
}

\author{
CARTOGRAPHIC ANALYSIS OF THE FRACTAL DIMENSION FOR THE SPACE- \\ TEMPORARY VARIATION OF RIVERS
}

\section{HECTOR LEANDRO GONZÁLEZ FERNÁNDEZ ${ }^{1}$ LUCÍA ARGÜELLES CORTÉS ${ }^{1}$, MICHAEL ÁLVAREZ GONZÁLEZ ${ }^{2}$}

${ }^{1}$ UNIVERSIDAD CENTRAL "MARTA ABREU” DE LAS VILLAS. Carretera a Camajuaní, Km 51⁄2, Santa Clara. Villa Clara, Cuba. E-mail: hgfernadez@uclv.cu; largue@uclv.edu.cu

${ }^{2}$ EMPRESA DE INVESTIGACIONES Y PROYECTOS HIDRÁULICOS DE VILLA CLARA (IPH VC). Ave. Libertadores 201 e/ Jesús Menéndez y Danielito, Santa Clara. Villa Clara, Cuba. E-mail: michael@vc.hidro.cu

Recibido: 12 de junio de 2019 / Aceptado: 20 de junio de 2019

\section{RESUMEN}

La Geometría Fractal es una nueva manera de ver la matemática en la naturaleza. En la actualidad tiene un notable aumento en cuanto a su desarrollo teórico y aplicado, mostrando ser idónea para describir los procesos naturales. Por tanto, en el presente trabajo se presenta como una herramienta para agilizar el proceso de actualización cartográfica aplicada a objetos naturales y antrópicos. A partir del algoritmo BoxCounting, se determina la dimensión fractal Box-Counting de los objetos naturales para utilizarla como un índice espacial de comparación que describe la capacidad que tiene un objeto de variar en el espacio a través del tiempo, y poder determinar así la necesidad de llevar a cabo la actualización cartográfica o no. Se verifica mediante un ejemplo real que el resultado obtenido es significativo tras la ocurrencia de eventos extremos y se infiere su utilidad para realizar una actualización cartográfica parcial de la zona afectada, lo cual reduce costos en el proceso cartográfico

Palabras claves: geometría fractal; dimensión fractal Box-Counting; cartografía digital.

\begin{abstract}
The Fractal Geometry is a new point of view in nature's mathematics. Now this theory has an increase in theoretic approach and applied researches. This kind of geometry is ideal to describe the natural processes in spatial complex distribution. Therefore, in the present work natural and anthropic objects are shown as a tool to speed up the cartography's update process. The Box Counting algorithm determines the fractal dimension index of the natural objects to use them like a comparison indicator as the capability that the object have to vary in the space through the time at the surface, and to be able to determine the cartography's update or not. It's verified by means of a real example that the obtained result is significant after the occurrence of extreme events and its utility is inferred to carry out a partial cartography's update of the affected area, which reduces costs in the cartographic process.
\end{abstract}

Key words: fractal geometry; Box-Counting fractal dimension; digital cartography. 


\section{INTRODUCCIÓN}

Los avances de la matemática vinculados a la geografía, así como la complejidad de los fractales y su presencia constante en el entorno antrópico, son problemas abordados y carentes de respuestas en diversas ramas del saber. La presencia de leyes físicomatemáticas que sustentan los fundamentos básicos de la cartografía digital posee sus orígenes en la geometría euclidiana, la cual se considera poco recomendable para abordar fenómenos del tipo multi-escala, dimensiones fraccionarias; por citar algún ejemplo al medir con más exactitud, la longitud de una costa aumenta. (Montesdeoca, 2005).

La geografía, desde sus principios ha estado relacionada de manera muy íntima con las matemáticas. Ambas han crecido de manera tan espectacular, que parecerían haberse alejado sin posibilidad de reconocerse mutuamente a punto de parecer dos ciencias independientes, pero a su vez dejando beneficios mutuos a ambas disciplinas. La recopilación de datos acerca de la distribución espacial de la superficie del planeta y su representación cartográfica ha sido una necesidad vital de cualquier sociedad. Los avances tecnológicos en el estudio científico de la superficie de la tierra, generó la necesidad de cartografiar nuevas regiones y cubrir mayores distancias.

El desarrollo del conocimiento y de la gestión de los recursos naturales, tales como geológicos, geomorfológicos, edáficos, ecológicos, hidrológicos o de uso del suelo, que tuvo sus inicios en el siglo pasado, ha continuado aprovisionándonos de nuevas necesidades de cartografías temáticas (Dueñas y Álvarez, 2010).

La Geometría Euclidiana pudiera ser la herramienta que permita relacionar la cartografía y la matemática. Entonces, “¿por qué a menudo se describe la geometría como algo frío y seco? Una de las razones es su incapacidad de describir la forma de una nube, una montaña, una costa o un árbol. Ni las nubes son esféricas, ni las montañas cónicas, ni las costas circulares..." (Mandelbrot, 1982). Las figuras comunes de la geometría clásica o euclidiana no son las más adecuadas para generar formas complejas como la hoja de un helecho o el perfil de una montaña.

Su limitación se debe a que tienden a perder su estructura cuando son ampliadas; un arco de círculo se transforma poco a poco en una recta y la superficie de una esfera se hace cada vez más plana.

La Geometría Fractal es bastante reciente en la matemática, su desarrollo se ha acelerado debido a su diversidad de aplicaciones en diferentes campos de la ciencia y la tecnología, gracias al desarrollo informático. Este tipo de geometría estudia figuras altamente irregulares generadas a través de procesos recursivos que tienen como característica fundamental la autosimilaridad y dimensión no entera. El primer aspecto significa que poseen alguna propiedad invariante bajo el cambio de escala, por ejemplo, a veces la rama de un árbol está compuesta por pequeñas ramas que tienen una forma muy parecida a la totalidad de la rama. El segundo significa que no posee las dimensiones usuales: uno, 
la de la línea; dos, el plano y tres, el espacio. Es decir, son figuras que pueden habitar en espacios intermedios; por ejemplo, encontrarse en el plano y en el espacio. Lo fundamental es reconocer cada una de las características del fractal y de su construcción (Aguilera, 1995).

En la actualidad es de suma importancia la actualización cartográfica, pues se considera que un mapa está envejecido cartográficamente en un periodo de 5 años según varios autores (Dalda, Cano, González, y Sánchez, 2003; Franco, 2000; Greenwalt and Shultz, 1962; Pellicer, 1980; Ramos, 2001). Por cuanto la cartografía nacional cubana a escala 1: 25000 que fueron creadas en una campaña fotogramétrica alrededor del 1980 con actualizaciones parciales hasta la fecha requiere de un proceso evaluación del envejecimiento de la información contenida en los mapas. Tomando en cuenta la desactualización de la cartografía cubana, se pretende crear métodos que ayuden a agilizar el proceso de actualización cartográfico a partir de herramientas matemáticas. Dada la evidente relación entre la Geometría Fractal -conocida también como la geometría de la naturaleza- y la geografía, no sería descabellado tratar de usarla en el proceso de actualización cartográfica, pues a primera vista debería ser una herramienta poderosa en estos trabajos. El poder identificar objetos naturales por su dimensión fractal y garantizar geometrías cartográficamente complejas acorde a los fenómenos naturales, implica una solución práctica a la cual se enfrentan los dibujantes y cartógrafos en la confección de planos y mapas actualmente.

Es por ello que el principal objetivo tratado en el presente trabajo se basa en proponer la dimensión fractal Box-Counting como índice decisor en el proceso de actualización cartográfica de objetos naturales a partir de imágenes aereosatelitales.

\section{MATERIALES Y MÉTODOS}

\section{- Geometría Fractal, Dimensión Box-Counting, Algoritmo Box-Counting}

La dimensión fractal en un sentido genérico es un número que sirve para cuantificar el grado de irregularidad y fragmentación de un conjunto geométrico o de un objeto natural. La dimensión fractal no es necesariamente entera (Rivera y López, 2011).

- Definición formal de fractal

Mandelbrot (1982) definió un "fractal" como un conjunto cuya dimensión de Hausdorff, es estrictamente mayor que su dimensión topológica.

El concepto de dimensión que usó Mandelbrot es una simplificación del de Hausdorff y corresponde exactamente a la definición establecida en 1958 por el matemático ruso Kolmogorov de la capacidad de una figura geométrica. Así, la capacidad de un conjunto $E$ se define como:

$$
d_{E}=\lim _{r \rightarrow 0}\left(\frac{\log (N(r))}{\log \left(\frac{1}{r}\right)}\right)
$$


donde si el conjunto $E$ es un subconjunto acotado de un espacio euclidiano pdimensional, entonces $N(r)$ es el mínimo número de cubos p-dimensionales de lado $r$ necesarios para cubrir $E$. Para un punto $N(r)=1$; para una línea es $N(r)=\frac{1}{r}$ y para una superficie es $N(r)=\frac{1}{r^{2}}$. Aplicando la expresión (2) se puede comprobar que $d_{E}=$ $0,1,2$ para un punto, una línea y una superficie plana, respectivamente.

La definición de Mandelbrot (1982) tiene el inconveniente que excluye conjuntos que por su autosemejanza debieran ser reconocidos como fractales. Se han propuesto otras definiciones de fractales, pero no existe todavía una, suficientemente general. En principio, se acuerda en no definir un fractal sino enumerar sus propiedades características, a saber:

- Un fractal tiene una estructura fina; esto es, mayor detalle en escalas arbitrariamente pequeñas;

- Un fractal es demasiado irregular para ser descrito con la Geometría Euclidiana tradicional, tanto local como globalmente;

- Con frecuencia, un fractal tiene una cierta forma de auto-semejanza, quizás aproximada o estadística;

- En muchos casos interesantes, el fractal se define en forma muy simple, por lo general, recursiva.

En el caso de figuras geométricas construidas por procesos recursivos, si $S$ es la cantidad de réplicas y $k$ es el factor de reducción, entonces $L=\frac{1}{k}$ es el factor de ampliación o de escala. A partir de la relación $S=(L)^{d}=\left(\frac{1}{k}\right)^{d}$ se tiene que la dimensión de Hausdorff $d$ viene dada de la manera siguiente:

$$
\log S=d \log L
$$

Despejando $d$ en la ecuación 2 quedaría:

$$
d=\frac{\log S}{\log L}
$$

\section{- Principales ejemplos. La curva que llena el plano. La curva de Koch.}

Existen curvas de relleno de dimensión n, la cual puede llenar una superficie, una de estas es la curva de Hilbert, la cual tiene un patrón y se va uniendo hasta completar el plano. Esta curva, es una muestra de cómo un objeto que naturalmente debería tener dimensión uno es capaz de llenar el plano y tener dimensión dos (véase la figura 1). 

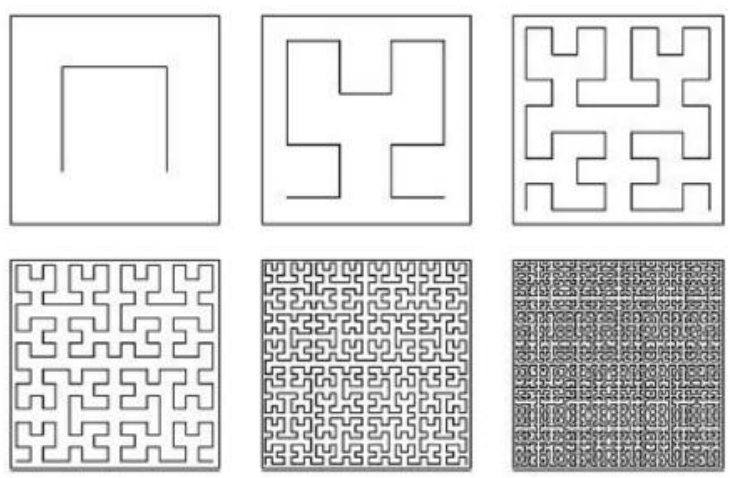

Figura 1. Ejemplo gráfico de la curva de Hilbert (Fuente: Valdés, 2016).

En 1904, el matemático sueco Koch descubrió una curva patológica, que obtenía como límite de una sucesión de polígonos. El carácter patológico del copo de nieve está dado por el hecho de cubrir un área finita; siendo su perímetro infinito. Además, es una curva continua que no tiene tangente en ningún punto. Este fractal está estrechamente vinculado al conjunto de Cantor y es, a su vez, el prototipo de una extensa familia de fractales, basados en la repetición de una transformación geométrica sumamente simple. (Sabogal y Arenas, 2011)

Se forma partiendo de un segmento unidad $K_{0}$, el cual se divide en tres partes iguales. La parte central se sustituye por dos segmentos del mismo tamaño que el eliminado, que junto con dicha parte anulada formaría un triángulo equilátero; se obtiene así la poligonal $K_{1}$. A continuación, se repite el proceso por cada segmento formado y se obtiene entonces una sucesión $\left(K_{j}\right) j \in \mathbb{N}$. El límite de esta sucesión, denotado $K$, se llama curva de Koch (véase la figura 2).

Nuevamente se percibe la autosimilitud de la figura límite, se detectan cuatro copias o réplicas $(S=4)$, y un factor de ampliación igual a tres $(\mathrm{L}=3)$, de modo que por su dimensión de Hausdorff (ecuación 3) quedaría la siguiente forma:

$$
D_{H}=\frac{\log 4}{\log 3} \approx 1.26186
$$

mientras que la dimensión topológica es $\mathcal{D T}=1$ (Barnsley, 2000)

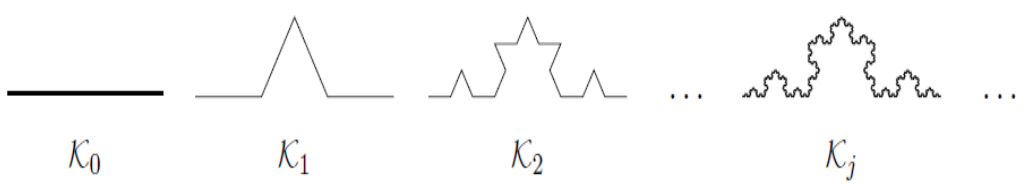

Figura 2. Ejemplo gráfico de la curva de Koch (Fuente: Valdés, 2016 y Domene, 2011). 
Se destaca en esta curva las características de autosimilitud y recursividad, pues a cada segmento $K_{0}$ que aparece se sustituye por $K_{1}$, y así cuando $n \rightarrow \infty$ aparece la figura límite $K_{j}$. Además $K_{2}$ se puede apreciar que está compuesta por tres veces $K_{1}$.

\section{- Algoritmo Box-Counting}

La dimensión fractal es presentada como una nueva forma de tratar aquellos objetos que no pueden ser analizados con la geometría euclidiana y que no son comunes encontrarlos en la matemática clásica. Los objetos autosimilares son de fácil manejo, la curva de Koch, el polvo de Cantor..., pero en la naturaleza existen objetos con alto grado de autosimilitud, que no son exactamente iguales, esto es lo que se denomina autosimilitud estadística (Mandelbrot, 1982). En estos casos también se puede hablar de una dimensión fractal en los objetos naturales.

El algoritmo de Box-Counting viene a generalizar en objetos naturales el método de cálculo de la dimensión fractal en objetos autosimilares, mediante generalizaciones apropiadas.

\section{- Dimensión de los objetos euclidianos}

A partir de un segmento de línea de longitud uno, se puede escalar el segmento por $\frac{1}{2}$ y observar 2 copias de longitud $\frac{1}{2}$, que cubren el segmento original. Se necesitan tres segmentos escalados por $\frac{1}{3}$, cuatro segmentos escalados por $\frac{1}{4}$, etc. Se debe tener en cuenta que $2=1 /(1 / 2), 3=1 /(1 / 3)$, y $4=1 /(1 / 4)$. En general, representando la escala por $r$ y el número de segmentos de escala $r$ por $N(r)$, tenemos la relación $N(r)=1 / r$ (véase la figura 3 )

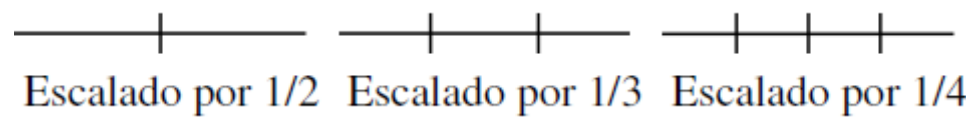

Figura 3. Ejemplo gráfico del proceso de escalado sobre una dimensión. Fuente: (Gutiérrez, 1998)

Para cubrir un cuadrado de lado 1 se necesitan 4 copias del cuadrado escalado por $1 / 2$ a lo largo de sus lados. Similarmente se necesitan 9 cuadrados escalados por $1 / 3,16$ cuadrados escalados por $1 / 4$, etc. Nótese que $4=(1 /(1 / 2))^{2}, 9=(1 /(1 / 3))^{2}$, y $16=$ $(1 /(1 / 4))^{2}$. En general, $N(r)=(1 / r)^{2}$ (véase la figura 4).

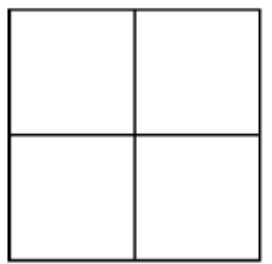

Escalado por $1 / 2$ Escalado por $1 / 3$

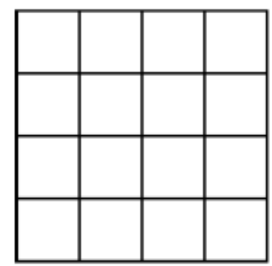

Escalado por $1 / 4$

Figura 4. Ejemplo gráfico del proceso de escalado sobre dos dimensiones Fuente: (Gutiérrez, 1998) 
Para cubrir un cubo de lado 1 se necesitan 8 copias de cubos escalados por $\frac{1}{2}$ a lo largo de todos sus lados. Similarmente se necesitan 27 cubos escalados por $\frac{1}{3}, 64$ cubos escalados por $\frac{1}{4}$, etc. Nótese que $8=(1 /(1 / 2))^{3}, 27=(1 /(1 / 3))^{3}$, y $64=$ $(1 /(1 / 4))^{3}$. En general, $N(r)=(1 / r)^{3}$ (véase figura 5$)$.

Para líneas, cuadrados y cubos se ha visto que hay una relación entre un factor de escala $r$ y el número $N(r)$ de copias de una forma similar, escalada por un factor $r$, necesitado para cubrir la forma original.

Representando por $d$ la dimensión Euclidiana de estas formas, la relación es: $N(r)=$ $(1 / r)^{d}$. Ahora, se puede afirmar que, si esta relación de escala se mantiene para una forma, entonces el valor de $d$ es la dimensión de dicha forma (véase la figura 5).

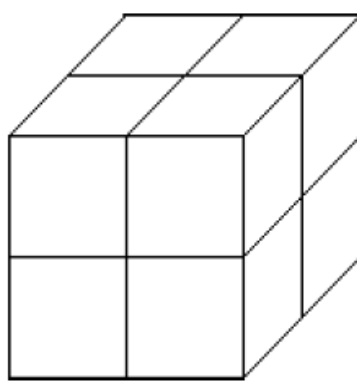

Escalado por $1 / 2$

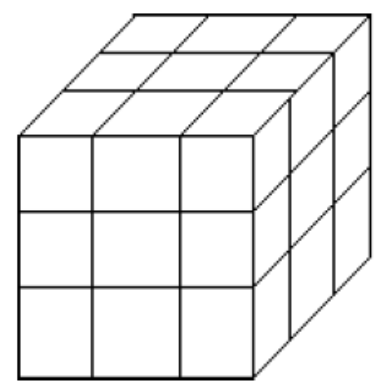

Escalado por $1 / 3$

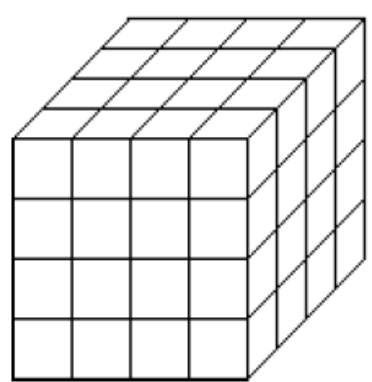

Escalado por $1 / 4$

Figura 5. Ejemplo gráfico del proceso de escalado sobre tres dimensiones Fuente: (Gutiérrez, 1998)

Se puede aplicar esta fórmula a cualquier objeto auto-similar, pero no a los fractales naturales, objetos físicos (no necesariamente exactamente auto-similares) que giran y giran tanto y en tantas escalas que se les puede considerar fractales. Para hacer frente a estos se necesita un método de aplicación más general para calcular las dimensiones.

\section{- Las cajas del Box-Counting}

Una malla es un conjunto de polígonos (triángulos, cuadriláteros, pentágonos), que definen una superficie en el espacio tal que, si $t_{i}$ y $t_{j}$ son dos elementos de una malla $T$, entonces: $t_{i} \cap t_{j}$ es un vértice, una arista común, o una cara común, o el conjunto vacío. Una malla tiene asociado un conjunto de elementos geométricos tales como: vértices, aristas y caras poligonales.

Para elementos finitos con una definición formal, una malla es un conjunto de nodos, o puntos representativos de un elemento, junto a sus relaciones de adyacencia [aristas, segmentos que unen nodos adyacentes de la malla (Domene, 2011; Meisel, \& Johnson, 1994). 
En el caso del Box-Counting se utilizarán mallas regulares compuestas por líneas paralelas y perpendiculares entre sí formando cuadrados o cajas (véase la figura 6).

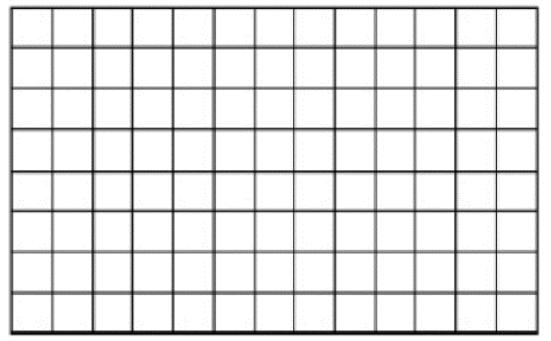

Figura 6. Ejemplo gráfico del espacio de análisis para poder aplicar la Dimensión Box-Counting Fuente: (Gutiérrez, 1998)

Si bien sabido es que los fractales naturales no contienen copias similares de sí mismos, ¿Cómo se puede medir su dimensión si no hay aparentemente un factor de escala? La respuesta es motivada por las observaciones euclidianas anteriores.

- Se cubre la forma con una malla de cajas y se cuenta el número de cajas que tocan cualquier parte de la forma.

- Se repite para mallas con cajas más y más pequeñas.

Se denota por $N(r)$ el número de cajas de la malla de lado $r$ tocando cualquier parte de la forma, se adopta la suposición, que

$$
N(r)=k\left(\left(\frac{1}{r}\right)^{d}\right)
$$

donde $k$ es la constante de proporcionalidad, 1 en los ejemplos previos. A partir de (4) tomando el $\log$ en ambos miembros, se obtiene la forma

$$
\log (N(r))=d \log \left(\frac{1}{r}\right)+\log (k)
$$

En la ecuación $5 d$ y $k$ son constantes, $\log (N(r))$ y $\log (1 / r)$ varían con $r$, de modo que tiene la forma: $y=m x+b$ de la ecuación de la línea recta con pendiente $d$ donde la variable dependiente sería $\log (N(r))$ y la independiente $\log (1 / r)$. Debido a que $d$ es interpretado como una dimensión en la escala original, se interpreta también como una dimensión en esta forma más general. Este $d$ se llama dimensión Box-Counting.

Para ello se realiza el proceso de conteo para las cajas ocupadas por el objeto del lado $r 1, r 2, \ldots, r N$. Para calcular $d$ desde estos box-counts (en este caso se hace referencia al proceso de conteo acorde a su redacción similar en idioma inglés según el algoritmo original): 
- $\quad$ se grafican $\operatorname{los}$ puntos $(\log (r 1), \log (N(r 1))), \cdots,(\log (r N), \log (N(r N)))$,

- luego se traza la línea del mejor ajuste a través de esos puntos y se calcula su pendiente.

Para fractales naturales la relación contenida en la ecuación 4, sólo es válida para una colección limitada de valores de $r$, denominada como el rango de escalamiento.

La dimensión Box-Counting puede ser calculada para fractales matemáticos y para más detalles son revelados con valores de $r$ más pequeños, tendiendo a cero, de modo que para fractales matemáticos se toma el límite:

$$
d=\lim _{r \rightarrow 0}\left(\frac{\log (N(r))}{\log \left(\frac{1}{r}\right)}-\frac{\log (k)}{\log \left(\frac{1}{r}\right)}\right)
$$

Debido a que $k$ es una constante, y $\frac{\log (k)}{\log \left(\frac{1}{r}\right)} \rightarrow 0$ cuando $r \rightarrow \infty$, (6) quedaría

$$
d=\lim _{r \rightarrow 0}\left(\frac{\log (N(r))}{\log \left(\frac{1}{r}\right)}\right)
$$

Para fractales naturales con un rango de escala limitada, este límite no es el enfoque más acertado debido a la no existencia de la autosimilitud exacta. De aquí que se pasa a trazar los puntos $(\log (1 / \mathrm{r}), \log (\mathrm{N}(\mathrm{r})))$, se hace necesario identificar el rango de escalamiento, y encontrada la pendiente de la recta que mejor se ajusta a través de los puntos en el rango de escalamiento se asume el mismo criterio propuesto por Camacho y Vásquez (2015) (consultar la figura 7) y otras experiencias similares alcanzadas por Nayak, Mishra, y Mohan (2018a, b) sobre imágenes a escalas de grises.

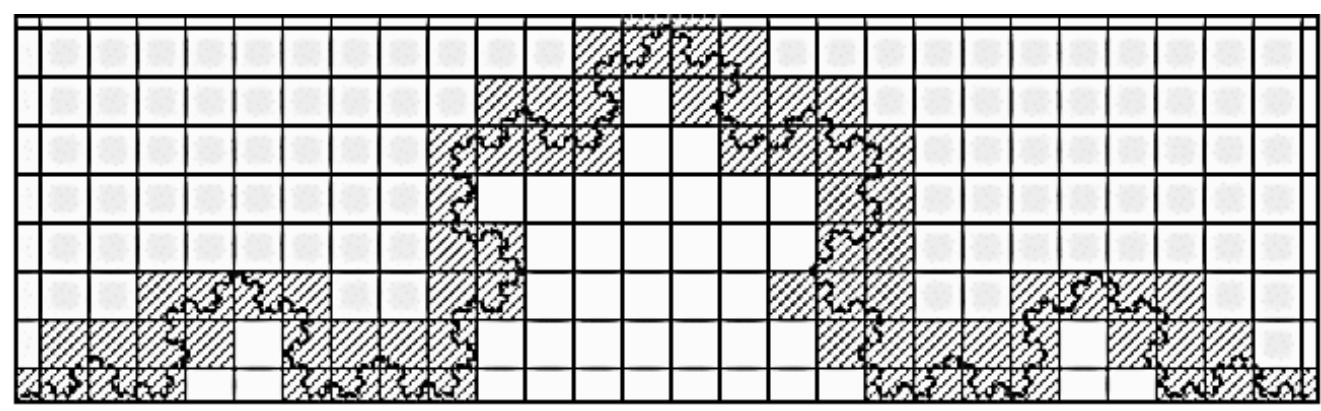

Figura 7. Representación gráfica del proceso de conteo de cajas por el método de Box-Counting. 


\section{- Análisis de la malla en el algoritmo Box-Counting}

En diferentes literaturas se trata el tema de la colocación de la malla para el cálculo de la dimensión fractal y la influencia que presenta sobre el resultado final (Bestehorn, 2018; Domene, 2011; Sabogal y Arenas, 2011; García, Mora, \& Redtwitz, 2017; López-Martín y Fraile-Jurado, 2011; Robinson, (n.d.)). Se conoce que la colocación de la malla sobre la figura en análisis es muy importante, pues la elección realizada trae consigo variaciones importantes en el cálculo de los valores de $N(r)$, a partir de cada tamaño de caja $r$ tomado (Loehle, 1994; Sabogal y Arenas, 2011). Una colocación exacta sobre la figura permite que la dimensión fractal no pase de dos, manteniendo el sentido de la dimensión usada en este trabajo como la capacidad que posee el objeto de llenar el espacio en que vive (consultar la figura 8).

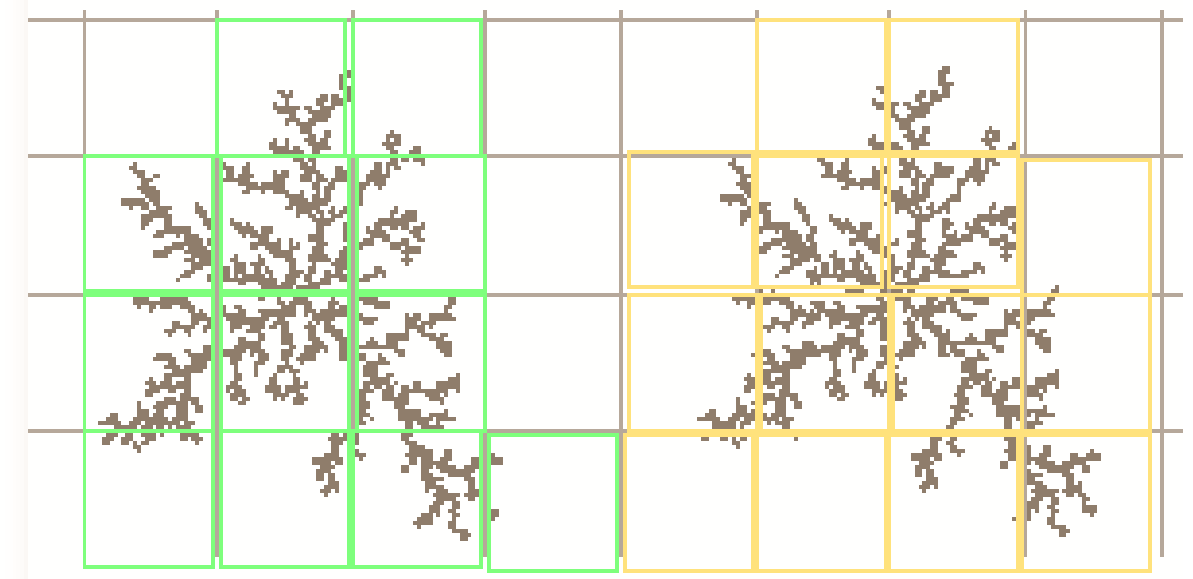

Figura 8. Representación gráfica de las diferentes colocaciones de la malla para aplicar el algoritmo Box-Counting sobre un mismo objeto de estudio (Fuente: Domene, (2011), p. 8).

Por otra parte, un tópico muy mencionado, pero poco trabajado en la literatura revisada es el de la elección del tamaño de la caja y el rango de escalamiento de estas para poder llevar a cabo el conteo necesario, siendo este criterio otro punto importante en la elaboración del algoritmo Box-Counting.

En caso alguno que fuera necesario valdría realizar la siguiente pregunta, ¿Cuál es el tamaño ideal para usar, y en qué razón debería disminuir dicho tamaño?

Para responder inicialmente esta problemática teórica, se comenzaría por presenta como objeto de análisis práctico para dar solución a esta pregunta, un fotograma de una imagen aerosatelital del río Bélico ubicado en la ciudad de Santa Clara (véase la figura 9). Este río posee la característica de ser confinado por las construcciones urbanas que se han desarrollado a todo lo largo de su ribera, elemento este que implica poca variabilidad 
espacial de su cauce en el tramo objeto de estudio. Además, se posee una amplia cartografía a diferentes escalas y años de mapificación para aplicar los elementos previamente comentados.

La Figura resultante de la zona geográfica en cuestión presenta un tamaño de $18,14 \mathrm{~cm}$ $\mathrm{x} 16,52 \mathrm{~cm}$.

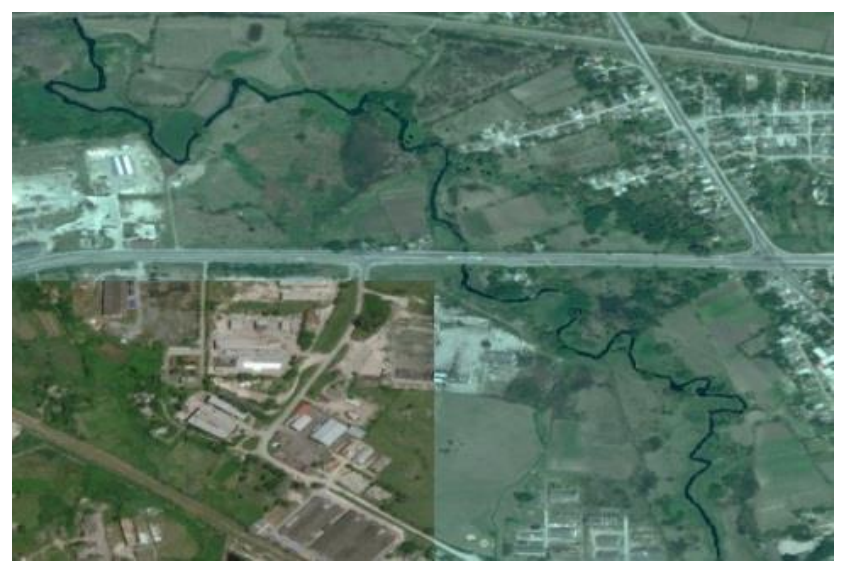

Figura 9. Imagen aero-satelital proveniente del SAS Planet de una sección del Río Bélico en la Ciudad de Santa Clara, Villa Clara, Cuba.

A partir del software computacional utilizado - Imaje $J$, software libre muy empleado para el cálculo de la dimensión fractal Box-Counting con un algoritmo ya implementado y que se utilizó como verificación de los resultados obtenidos del código creado por los autores implementado con una cuenta gratuita en la nube del software Wolfram Mathematics- se realizó un trabajo de edición de imagen como preparación al uso de los algoritmos antes mencionados, donde se transformó la imagen aerosatelital en una imagen binaria (blanco-negro), es decir, el objeto a analizar, el río, se coloreó de negro y el resto de la imagen se transformó en color blanco (véase la figura 10).

Para llevar a cabo el algoritmo Box-Counting se utilizaron los pixeles de la imagen como malla, tomando los píxeles de color negro como las cajas que se superponían sobre el río. Por tanto, se realizaron varias pixelaciones de la imagen, desde 5 píxel por centímetro, que equivale a 25 píxeles por centímetro cuadrado, hasta 1000 píxel por centímetro que equivale a 1000000 de píxeles por centímetro cuadrado. Como resultado se obtuvo la siguiente relación: 


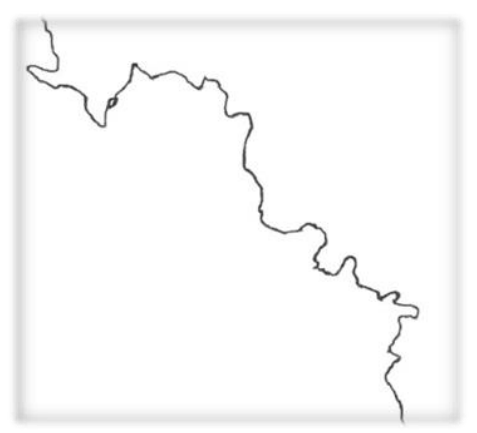

Figura 10. Transformación binaria de la imagen aero-satelital.

Tabla 1. Conteo de los Píxeles por centímetro cuadrado de las diferentes dimensiones o escalado asumido en el presente estudio

\begin{tabular}{|l|l|l|l|l|l|l|}
\hline $\begin{array}{c}\text { Píxel por } \\
\mathbf{c m}\end{array}$ & \multicolumn{1}{|c|}{$\begin{array}{c}\text { Tamaño en } \\
\mathbf{c m}\end{array}$} & \multicolumn{1}{|c|}{$\begin{array}{c}\text { Tamaño en } \\
\text { píxeles }\end{array}$} & \multicolumn{1}{|c|}{$\mathbf{r}$} & \multicolumn{1}{|c|}{$\log \mathbf{1 / r}$} & \multicolumn{1}{|c|}{$\mathbf{N}(\mathbf{r})$} & \multicolumn{1}{|c|}{$\log \mathbf{N}(\mathbf{r})$} \\
\hline $5 \mathrm{ppcm}$ & $18,14 \times 16,52$ & $\begin{array}{l}\{\text { C. van Westen, } \\
1997 \# 132\}\end{array}$ & 0,2000000 & 0,69897000 & 677 & 2,830588669 \\
\hline $10 \mathrm{ppcm}$ & $18,14 \times 16,52$ & $\{181,165\}$ & 0,1000000 & 1,00000000 & 29865 & 4,475162519 \\
\hline $25 \mathrm{ppcm}$ & $18,14 \times 16,52$ & $\{453,412\}$ & 0,0400000 & 1,39794001 & 86636 & 4,937698393 \\
\hline $50 \mathrm{ppcm}$ & $18,14 \times 16,52$ & $\{907,826\}$ & 0,0200000 & 1,69897000 & 749182 & 5,874587334 \\
\hline $100 \mathrm{ppcm}$ & $18,14 \times 16,52$ & $\{1814,1652\}$ & 0,0100000 & 2,00000000 & 2996728 & 6,476647326 \\
\hline $150 \mathrm{ppcm}$ & $18,14 \times 16,52$ & $\{2721,2478\}$ & 0,0066666 & 2,17609126 & 6742638 & 6,828829844 \\
\hline $200 \mathrm{ppcm}$ & $18,14 \times 16,52$ & $\{3628,3304\}$ & 0,0050000 & 2,30103000 & 11986912 & 7,078707317 \\
\hline $500 \mathrm{ppcm}$ & $18,14 \times 16,52$ & $\{9070,8260\}$ & 0,0020000 & 2,69897000 & 74918200 & 7,874587334 \\
\hline $600 \mathrm{ppcm}$ & $18,14 \times 16,52$ & $\{10884,9912\}$ & 0,0016666 & 2,77815142 & 10788220 & 8,03294982 \\
\hline $700 \mathrm{ppcm}$ & $18,14 \times 16,52$ & $\{12698,11564\}$ & 0,0014285 & 2,8450981 & 14683967 & 8,16684340 \\
\hline $800 \mathrm{ppcm}$ & $18,14 \times 16,52$ & $\{14512,13216\}$ & 0,0012500 & 2,9030899 & 19179059 & 8,2828273 \\
\hline $1000 \mathrm{ppcm}$ & $18,14 \times 16,52$ & $\{18140,16520\}$ & 0,0010000 & 3,0000000 & 29967280 & 8,47664732 \\
\hline
\end{tabular}

A partir de esta Tabla 1, el siguiente paso del algoritmo a implementar fue el de su representación gráfica logaritmo-logaritmo de los pares ordenados $\left(\log \frac{1}{r}, \log N(r)\right.$ ), a los cuales se les calcula la recta de regresión y cuya pendiente es la dimensión fractal Box-Counting (véase la figura 11).

Luego de llevado a cabo el proceso, se obtiene como resultado la recta $y=2,2586 x+$ 1,8121 con un error cuadrático medio de 0.98. Por tanto, la dimensión fractal Box- 
Counting para el tramo parcial objeto de estudio del río Bélico sería 2,2586. Ahora si se toma en cuenta la definición con que se está trabajando, -la capacidad del objeto para llenar el espacio en que vive-, ¿cómo es posible que la dimensión entonces sea mayor que 2? No es posible, se está incurriendo en un error teórico. Pero, ¿dónde está ese error?

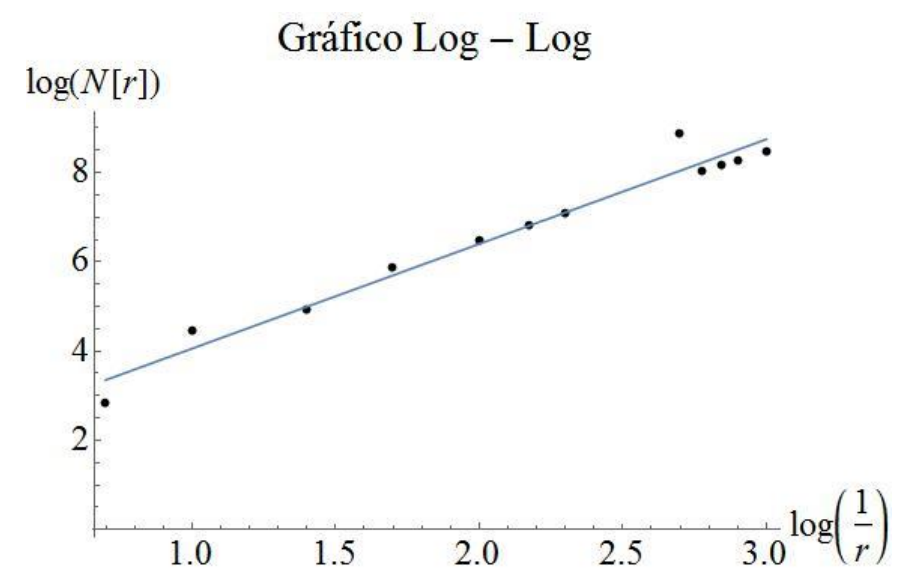

Figura 11. Recta de regresión y de la dimensión fractal Box-Counting.

Las mallas utilizadas para este proceso, como se aprecia en la Tabla 1, llevan a disminuir el tamaño de la caja, pero de forma desproporcionada, -no existe un valor $k$ de proporción-, por lo que el rango de escala se ve afectado y el algoritmo no devuelve el resultado correcto.

A partir de estos resultados se llevaron a cabo modificaciones en el algoritmo. Se realizó una primera pixelación de la imagen donde el tamaño de la caja se tomó como la unidad. Luego cada nueva pixelación sería el doble de la anterior, lo que conllevaría a que la caja siguiente sería la mitad de la anterior. Por tanto, los tamaños de caja fueron los siguientes:

Tabla 2. Proceso de pixelación de la imagen luego de modificado el algoritmo Box-Counting.

\begin{tabular}{|l|l|l|c|c|c|}
\hline $\begin{array}{c}1 \text { ra } \\
\text { pixelación }\end{array}$ & \multicolumn{1}{c|}{$\begin{array}{c}\text { 2da } \\
\text { pixelación }\end{array}$} & $\begin{array}{c}\text { 3ra } \\
\text { pixelación }\end{array}$ & $\begin{array}{c}\text { 4ta } \\
\text { pixelación }\end{array}$ & $\begin{array}{c}5 \text { ta } \\
\text { pixelación }\end{array}$ & $\begin{array}{c}6 \text { ta } \\
\text { pixelación }\end{array}$ \\
\hline 1 unidad & $1 / 2$ unidad & $1 / 4$ unidad & $1 / 8$ unidad & $1 / 16$ unidad & $1 / 32$ unidad \\
\hline
\end{tabular}

Con estas nuevas mallas, se procedió a realizar de nuevo todo el proceso del algoritmo, es decir, el cálculo de los nuevos valores de $N(r)$ a partir de los valores de $r$ de la Tabla 2, donde se obtuvieron los resultados presentados en la figura 12: 


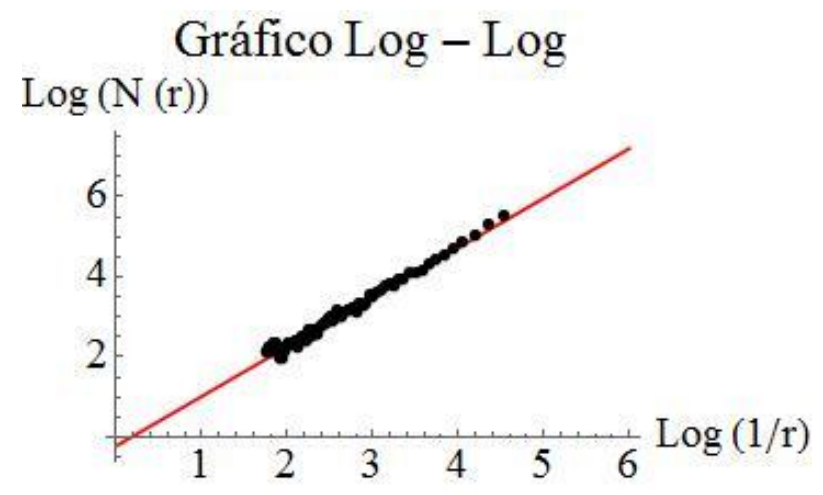

Figura 12. Representación del gráfico log-log y su recta de regresión.

La ecuación de la recta de regresión obtenida es: $y=-0.41640+1.29381 x$ con un error cuadrático medio de $\varepsilon=0.99$ en el ajuste lineal del gráfico. Por tanto, la dimensión fractal Box-Counting sería 1,20854. Este resultado es correcto y verificado por los dos programas computacionales mencionados anteriormente. Además, teóricamente es correcto, pues la dimensión fractal es menor de dos, acorde a la dimensión del plano donde "vive" el río analizado (dígase el lugar donde "vive" el objeto el ambiente geométrico-espacial donde se encuentra, ejemplo, el río está en el suelo que se toma como un plano, y un árbol está en el espacio, o sea tiene tres dimensiones).

Para ello se propone la ejecución de los procesos investigativos acorde a la figura 1. Dónde se realiza un análisis de correlación entre los niveles de agua del embalse y el comportamiento de las patologías de filtraciones, además de establecer comparaciones empíricas a partir de datos correlativos en lo referente al comportamiento de las deformaciones provenientes de las mediciones geodésicas en el dominio del tiempo.

En un segundo momento se desarrollan las simulaciones de los diferentes escenarios probables por medio de técnicas estocásticas (aleatorias), indizando el grado de incertidumbre asociado a la posibilidad o no de ocurrencia del fenómeno, además de evaluar el comportamiento tenso-deformacional de la estructura asociado a los probables escenarios de ocurrencia por medio de la geo-visualización de las deformaciones correspondientes a cada marca geodésica de control en la cortina del embalse.

\section{DISCUSIÓN DE LOS RESULTADOS ALCANZADOS}

- Análisis del Río Bélico

A partir de datos cartográficos públicos pertenecientes a la provincia de Villa Clara, Cuba a escala 1: 50000 (véase la figura 12) y de las imágenes obtenidas mediante el uso del software libre SAS Planet, se inició el proceso de demostración de la hipótesis planteada en el objetivo de la presente investigación. En un primer momento se analizó un tramo del río Bélico, situado en la ciudad de Santa Clara, Villa Clara, Cuba. 
Para ello fueron considerados diferentes momentos en el tiempo, y a diferentes escalas, se realizó el análisis de la dimensión fractal usando el algoritmo Box-Counting según se muestra a continuación:

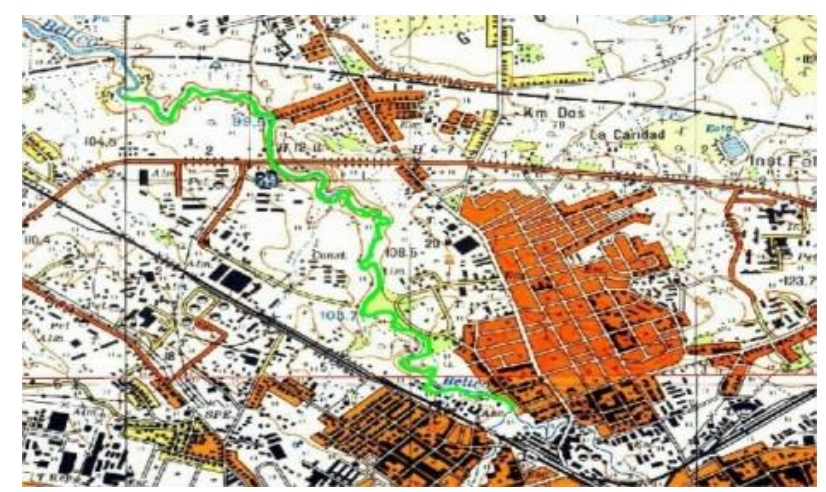

Figura 12. Imagen parcial del plano a escala 1:25 000 de la Ciudad de Santa Clara perteneciente al año 1984.

A partir de la imagen base se realiza un procesamiento de la imagen para obtener los datos necesarios para poder utilizar el algoritmo Box-Counting (véase la figura 13-15). A partir de ahora se utilizará similar método de trabajo, tomando los siguientes resultados como puntos comparativos con el momento inicial del tramo del rio para diferentes momentos en el tiempo y cartografías fuentes según la tabla 3.

Tabla 3. Análisis Río Bélico a diferentes escalas y en diferentes momentos en el tiempo.

\begin{tabular}{|c|c|c|c|}
\hline Año & Escala & Dimensión Fractal B-C & $\begin{array}{c}\text { Error cuadrático } \\
\text { medio }\end{array}$ \\
\hline 1984 & $1: 25000$ & 1,23578 & 0,99 \\
\hline 1990 & $1: 2000$ & 1,24834 & 0,97 \\
\hline 2014 & $1: 10000$ & 1,21749 & 0,98 \\
\hline 2018 & Imagen Satelital & 1,29381 & 0,99 \\
\hline
\end{tabular}
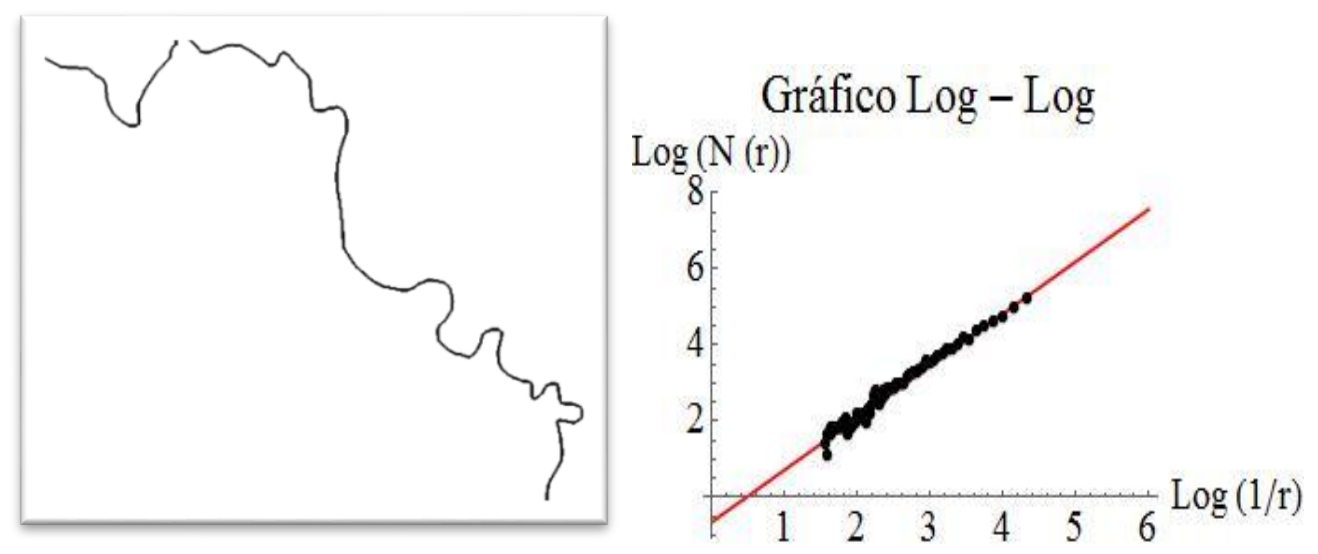

Figura 13. Imagen binaria a partir de un tratamiento de imagen de la cartografía contenida en la figura 9 y su representación del gráfico log-log de su recta de regresión 

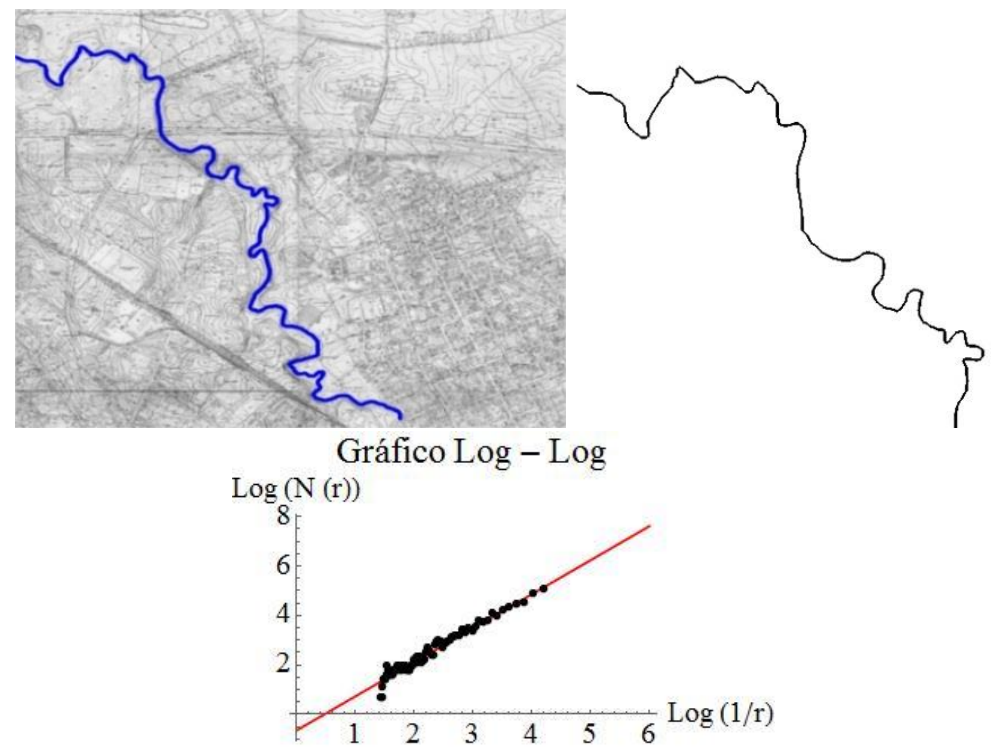

Figura 14. Vectorización realizada sobre el mismo tramo del Río Bélico en un plano a escala 1: 2000 de la Ciudad de Santa Clara confeccionado en el año 1990 y su recta de regresión.

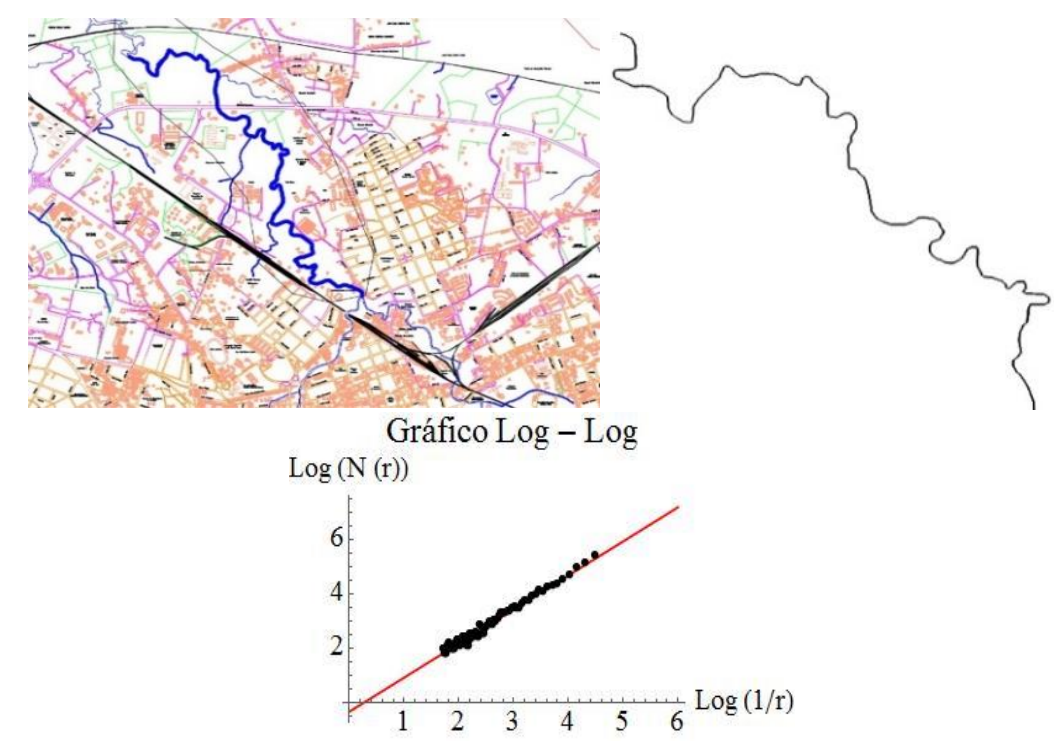

Figura 15. Vectorización realizada sobre el mismo tramo del Río Bélico en un plano a escala 1: 10000 de la Ciudad de Santa Clara confeccionado en el año 2014 y su recta de regresión.

Se puede apreciar que todos los valores obtenidos se encuentran entre 1,2 y 1,3; por tanto, promediando estos valores obtenemos 1,24885 con una desviación estándar de 0,033 ; lo cual podemos asumir como la dimensión fractal del Río Bélico. Pero a qué se debe la pequeña variación en los dígitos después de la coma. A pesar de ser un río confinado 
dentro de la ciudad con el pasar de los años sufre leves cambios (véase la figura 16), lo que queda ejemplificado en dicha variación numérica.

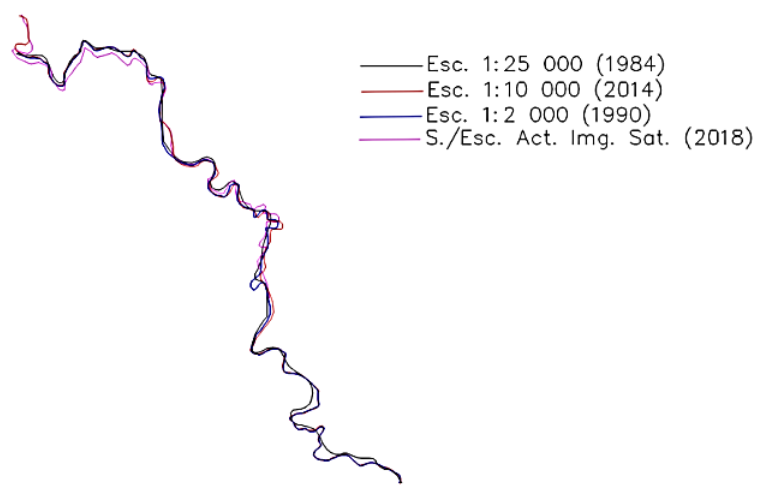

Figura 16. Superposición de vectores realizada partir de las diferentes escalas y diferentes años de una misma sección geográfica del Río Bélico en la Ciudad de Santa Clara, Villa Clara, Cuba.

\section{- Análisis del Río Sagua la Grande}

El río Sagua la Grande atraviesa toda la provincia de Villa Clara, Cuba. Nace en la Sierra del Escambray y desemboca al norte de la isla y en su recorrido se encuentran varios embalses, incluido el segundo de mayor volumen de almacenamiento a nivel nacional conocido como Alacranes. Es uno de los ríos de mayor caudal de la isla y su nombre se debe al municipio Sagua la Gande de la provincia previamente mencionada.

Este análisis pretende probar la variación que existe en la dimensión fractal BoxCounting del objeto lineal al existir una gran variación de este en el plano.

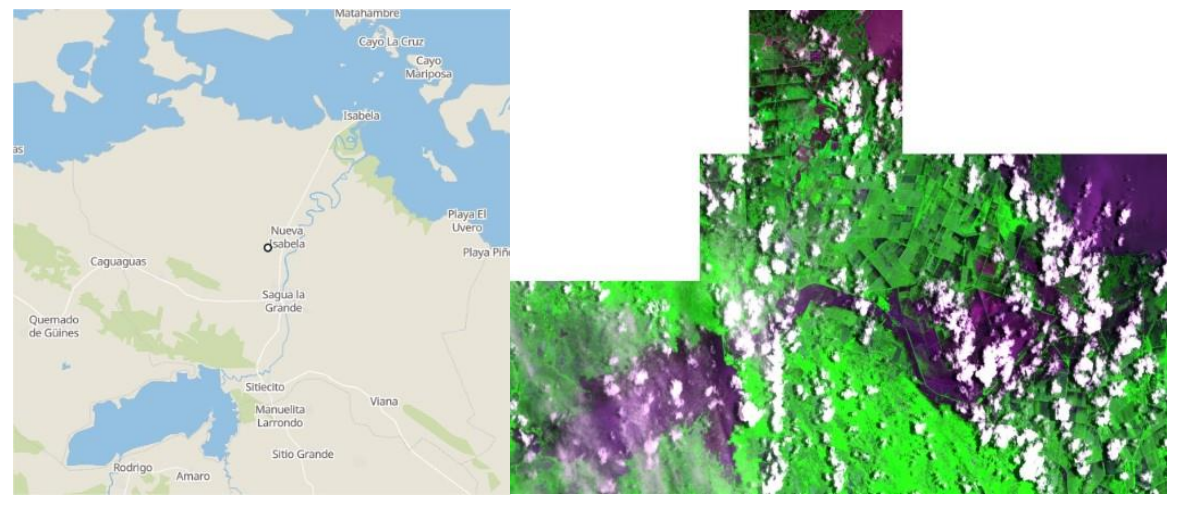

Figura 17. Representación cartográfica del río Sagua la Grande (Fuente: Earth Observing System [EOS] ( Copyright 2017).

Manteniendo las directrices de trabajo utilizadas anteriormente, se realiza el procesamiento de la imagen y se obtiene como resultado: 

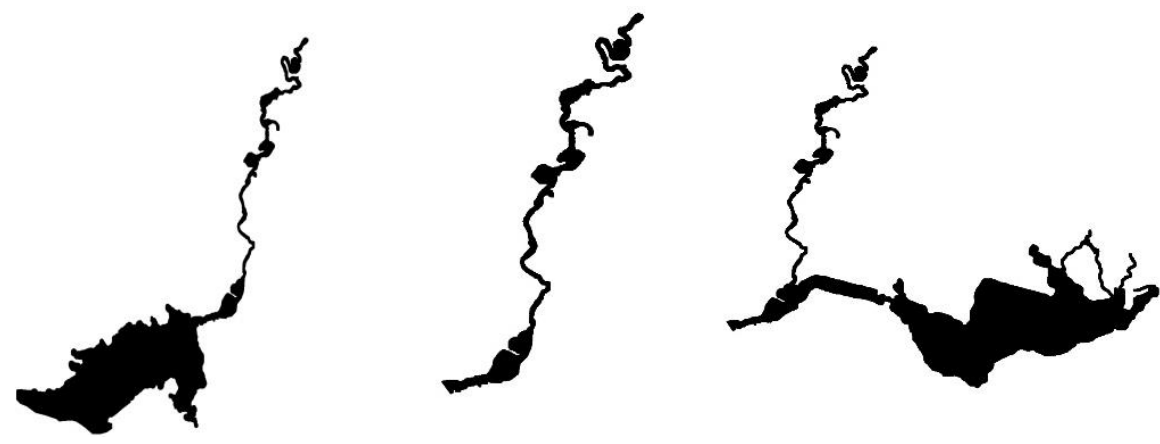

Figura 18. Resultado del procesamiento digital (desde la izquierda a la derecha) desde el Embalse Alacranes y el río Sagua la Grande en su estado natural (izquierda), la extracción del río desde el embalse (centro) y el desborde del río en el caso de un evento extremo de lluvias durante la tormenta tropical Alberto en el 2018 (derecha).

Al realizar el análisis de la dimensión fractal Box-Counting, los resultados son mostrados en la siguiente figura y posterior tabla.
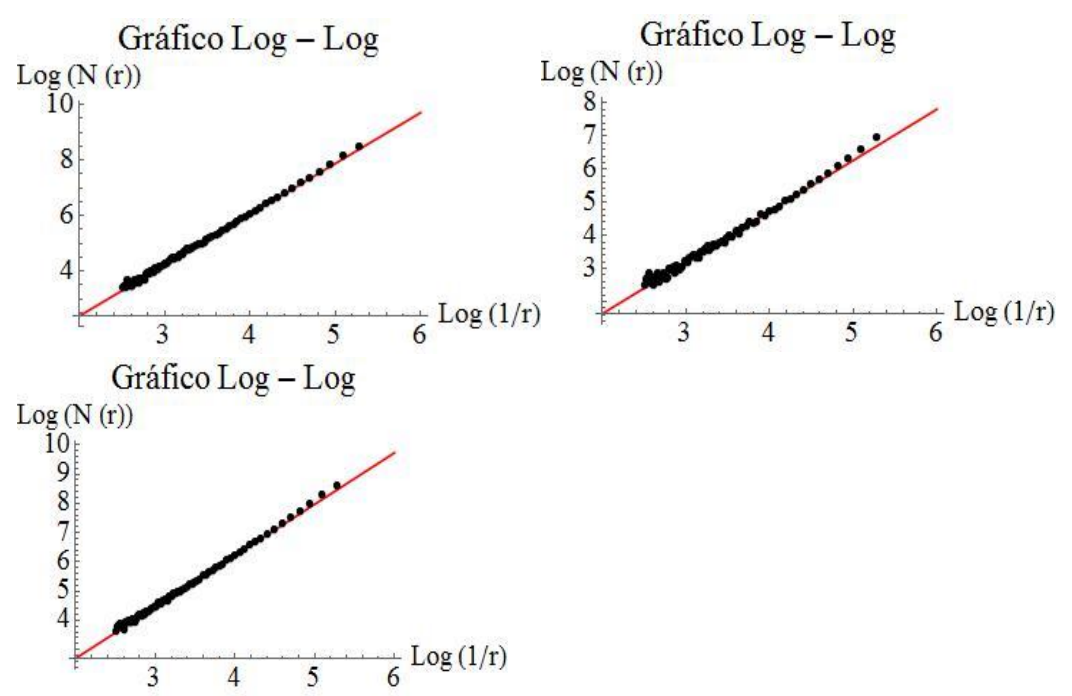

Figura 19. Gráficos log-log del procesamiento de las imágenes de la figura 18 manteniendo el orden.

Tabla 4. Análisis de la dimensión fractal del Río Sagua la Grande a partir de la figura 18.

\begin{tabular}{|l|c|c|}
\hline \multicolumn{1}{|c|}{ Figura } & $\begin{array}{c}\text { Dimensión } \\
\text { Fractal B-C }\end{array}$ & $\begin{array}{c}\text { Error cuadrático } \\
\text { medio }\end{array}$ \\
\hline $\begin{array}{l}\text { Embalse Alacranes y Río Sagua la Grande en estado } \\
\text { natural. }\end{array}$ & 1,23578 & 0,99 \\
\hline Río Sagua la Grande sin la presa Alacranes.. & 1,82900 & 0,99 \\
\hline $\begin{array}{l}\text { Río Sagua la Grande desbordado a partir de las lluvias } \\
\text { durante la tormenta tropical Alberto en el 2018. }\end{array}$ & 1,75669 & 0,99 \\
\hline
\end{tabular}


Se aprecia que el resultado obtenido es consecuente con la idea de que a mayor dimensión fractal, mayor espacio ocupa el objeto lineal en el terreno, siendo consecuente con la definición de dimensión fractal utilizada y en comparación con el valor de desviación estándar del caso de estudio previo, ya que supera alrededor de 10 veces su valor $(0,324)$. Este proceso de variación implica una nueva distribución espacial del objeto lineal cartográfico que por necesidad requiere ser actualizado en el plano o serie de planos que son interés particular de la presente investigación.

La secuencia de análisis responde a un algoritmo orientado al estudio de la variación cartográfica de los objetos contenidos en un plano para poder determinar cronológicamente la necesidad de actualización cartográfica de un mapa, sea de forma parcial o total, a partir de los principales objetos actuantes que modifican el relieve.

\section{CONCLUSIONES}

Acorde a los resultados previamente comentados se proponen las siguientes conclusiones:

1. El proceso de selección del escalado de la malla en el algoritmo Box-Counting para calcular la dimensión fractal implica una relevante importancia en la veracidad del resultado adecuado a la geometría cartográfica y su variación espacio-temporal.

2. La dimensión fractal media calculada por el Método Box-Counting del Río Bélico ubicado en la ciudad de Santa Clara, Cuba, es de 1,24885; este valor contiene la respuesta cartográfica de su variación espacio-temporal contenida en el coeficiente de desviación estándar para 0,033 como un coeficiente de sensibilidad para el mismo objeto en diferentes fechas cronológicas. Para el caso del río Sagua la Grande, se evidencia cómo varía significativamente su dimensión fractal ante la ocurrencia de un evento hidro-meteorológico extremo que altera cartográficamente la geometría del cauce del río y en consecuencia la necesidad de una actualización cartográfica de este objeto de estudio, dado que el coeficiente de desviación estándar resulta ahora 0,324.

3. El valor de la dimensión fractal de los objetos estudiados fue caracterizada por una diferencia significativa en el caso de una variación cartográfica en la superficie del terreno ante la ocurrencia de eventos meteorológicos extremos y de una diferencia mínima en correspondencia a objetos cartográficos lineales confinados o de menor sensibilidad a los cambios espaciales ante eventos meteorológicos extremos.

4. La dimensión fractal calculada por el método Box-Counting puede ser utilizada al estudio de las variaciones espaciales de los objetos cartográficos de un mapa acorde a la necesidad de estimar cuando realizar una actualización cartográfica parcial o total de los objetos cartográficos que modifican el relieve. 


\section{REFERENCIAS BIBLIOGRÁFICAS}

Aguilera, N. (1995). Un paseo por el jardín de los fractales. Buenos Aires: Red Olímpica.

Ana García Domene. (2011). Box Counting. Matemáticas II, Ingeniería multimedia. Disponible en: www.fractal.art.pl

Barnsley, M. (2000). Fractals everywhere (2. ed., [Nachdr.]). San Diego, Calif.: Morgan Kaufmann [u.a.].

Bestehorn, M. (2018). Fraktale Dimension: Box Counting. Physik Online. doi:10.1515/physiko.17.21.

Camacho, R. y Vásquez, M. (2015). Geometría fractal, Teoría del caos, y sus aplicaciones en la Industria Petrolera, Vol. 55, No. 12, p. 12.

Dalda M, Cano M, González F, y Sánchez J. (2003). IV Curso GPS para Geodesia y Cartografía. Colombia: AECI.

Dueñas R, y Álvarez M. (2010). Teledetección y Sistemas de Información Geográfica para Ingenieros Agropecuarios y Mecanizadores Apuntes para un Folleto-Texto (Primera Edición). Cuba: Samuel Feijó.

Franco J. (2000). Nociones de Topografía, Geodesia y Cartografía. Barcelona.

García, G., Mora, G., \& Redtwitz, D. A. (2017). Box-Counting dimension computed by $\alpha$-dense curves. Fractals, 25(05), 1750039. doi:10.1142/s0218348x 17500396

Greenwalt, C. and Shultz, M. (1962). Principles of error theory and cartographic applications.

Gutiérrez, José Manuel. (1998). SISTEMAS NO LINEALES. CONCEPTOS, ALGORITMOS Y APLICACIONES (p. 28). Presentado en V CONFERENCIA NACIONAL DE CIENCIAS DE LA COMPUTACIÓN, Potosí.

Loehle, C. (1994). Rescaled box counting for the topological analysis of spatial data. doi:10.2172/10142283

López-Martín. M. y Fraile-Jurado, P. (2011). Análisis preliminar del cálculo de la dimensión fractal de diferentes tipos de costa en el litoral andaluz, p. 40.

Mandelbrot, B. (1982). The Fractal Geometry of Nature. (1ra Edition). New York: W. H. Freeman and Company.

Meisel, L. V., \& Johnson, M. A. (1994). Numerical Box-Counting and Correlation Integral Multifractal Analysis. doi:10.21236/ada282902

Nayak, S. R., Mishra, J., \& Mohan Jena, P. (2018a). Fractal analysis of image sets using differential box counting techniques. International Journal of Information Technology, 10(1), pp. 39-47.

Nayak, S. R., Mishra, J., \& Jena, P. M. (2018b). Fractal Dimension of GrayScale Images. In Progress in Computing, Analytics and Networking (pp. 225-234). Springer, Singapore.

Montesdeoca Pérez, Pablo. (2005). Longitud y Área de Curvas Fractales. Dimensión Fractal. Longitud y Área de Curvas Fractales. Dimensión Fractal, 15.

Pellicer, J. (1980). Cartografía (Primera edición). Cuba: Pueblo y Educación.

Ramos, A. (2001). Los Sistemas de Información Geográfica (SIG) Cartografía. Presentado en Cátedra de Geografía y Geodesia, Cuba.

Rivera, E. y López, R. (2011). Geometría fractal y transformada de Fourier. (Vol. XVI). Colombia: Scientia ET Technica.

Robinson, J. C. (n.d.). Box-counting dimension. Dimensions, Embeddings, and Attractors, pp. 31-40. doi:10.1017/cbo9780511933912.005.

Sabogal, S. and Arenas, G. (2011). Una introducción a la geometría fractal. Universidad Industrial de Santander Bucaramanga.

Valdés, P. (2016). Introducción a la Geometría Fractal. Chile: Universidad del Bío-Bío. Sistema de Bibliotecas - Chile. 